\title{
Comparación entre controles hápticos y tradicionales en educación y entrenamiento para RV
}

\author{
Comparison between haptics and traditional controls for VR \\ education and training
}

\author{
Pablo Figueroa $^{1}$ (D), Fabián Medina Cortés ${ }^{1}$ \\ ${ }^{1}$ Universidad de los Andes, Bogotá D.C., Colombia \\ pfiguero@uniandes.edu.co,df.medinac@uniandes.edu.co
}

(Recibido: 2 Mayo 2020; aceptado: 20 Septiembre 2020; Publicado en Internet: 1 Diciembre 2020)

\begin{abstract}
Resumen. La Realidad Virtual (RV) como tecnología generadora de ambientes inmersivos actualmente presenta una notable expansión, y se ha presenciado en múltiples investigaciones en diversos campos de estudio. Reconociendo las virtudes que aporta esta tecnología, como el aumento en motivación y en la efectividad del aprendizaje (por comprender un mayor nivel de inmersión y satisfacción en las actividades realizadas) en los ámbitos educativos y de entrenamiento, se propone evaluar cuál es la configuración de controles (hápticos o tradicionales) más pertinente para mejorar el desempeño y la percepción de usabilidad en los ámbitos mencionados. Así pues, este articulo presenta detalles correspondientes a la elaboración de un prototipo que permita la finalización de tareas básicas para fines educativos y de entrenamiento, en un ambiente de realidad virtual. En este documento nos centramos en las pruebas realizadas al prototipo para definir la interacción más usable a criterio de los participantes del experimento.
\end{abstract}

Palabras clave: Háptico, Usabilidad, Controles, Realidad virtual, Entrenamiento.

Abstract. Virtual Reality (VR) as an immersive environments generator currently presents a notable expansion and has made an appearance on multiple investigations concerning various study fields. Acknowledging the virtues presented by this technology, such as a rise in both motivation and learning effectiveness (by bringing an increased immersion and satisfaction levels on performed activities) on educational and training scopes, it's been proposed to assess which control configuration (haptics or traditional) is more relevant to improve performance and usability on the mentioned scopes. Therefore, this article presents corresponding details to elaborating a prototype that allows the accomplishment of basic tasks for educational and training purposes in a virtual reality environment. In this document, we focus on the prototype's tests to determine the most usable interaction based on the criteria of the experiment's participants.

Keywords: Haptics, Usability, Controls, Virtual reality, Training.

Tipo de artículo: Artículo de investigación.

\section{Introducción}

Uno de los mayores retos que afronta la ingeniería en áreas como la computación aplicada, es ofrecer interfaces usables y de calidad con el fin de generar la satisfactibilidad de los usuarios; más aún en tecnologías que han tenido grandes aportes en investigación en los últimos años como lo son la realidad virtual y aumentada (Ma et al., 2014). Algunas áreas que se han visto beneficiadas por los diferentes aportes de estas tecnologías son las medicina (Boejen \& Grau, 2011), arquitectura (Boychenko, 2017), educación (Yilmaz \& Goken, 2016), entre otros. Las áreas de educación y entrenamiento con el apoyo de la realidad virtual serán el tema central de este estudio.

El prototipo construido en este estudio planea realizar una comparación con el fin de evaluar criterios de usabilidad entre interfaces que implementen controles hápticos y tradicionales. De esta manera, por medio de un experimento concluir qué tipo de controles son más adecuados para manejar interfaces inmersivas. Para los controles hápticos se usan sensores Phidgets y para los tradicionales un control de mando de consola. Además de lo anterior, las personas serán sometidas a pruebas en las cuales deberán completar tareas en interfaces con las mismas condiciones y los dos tipos de controles mencionados; posteriormente diligenciarán encuestas que permitan realizar la comparación de los tipos de controles. Por último, el 
objetivo generalizado del proyecto es realizar un prototipo para educación/entrenamiento con realidad virtual que garantice ser superior en términos de usabilidad.

\section{Estado del arte}

Vamos a considerar el estado del arte desde diversas facetas, primero observando cómo la realidad virtual ha sido utilizada en educación y luego revisando en particular aplicaciones de RV que utilicen mecanismos hápticos.

\subsection{Evaluación por pares}

La Realidad Virtual es una nueva tecnología que consiste en la generación de ambientes inmersivos en los cuales el usuario tiene la sensación de estar físicamente presente, y puede interactuar con ellos en tiempo real. Hoy en día, se observa una notable expansión de esta tecnología y múltiples investigaciones en diversos campos (Boejen \& Grau, 2011; Boychenko, 2017; Yilmaz \& Goken, 2016).

En el campo de educación, el uso de esta tecnología es la oportunidad perfecta para generar en los estudiantes motivación y aprendizaje efectivo tal como se plantea en (Al-Shamaileh, 2014), desde el punto de vista psicológico (Wang \& Li, 2012), la sensación de estar en un lugar y presenciar una experiencia significativa genera valor, ya que da la posibilidad de utilizar dichos contextos virtuales como potentes herramientas que ayuden a modificar conductas, pensamientos, experiencias y emociones.

Estudios con sistemas virtuales para entrenamiento de rescates de emergencia y habilidades avanzadas de manufactura (Li et al., 2005; Watanuki \& Kojima, 2006) muestran cómo es posible generar interacciones naturales que generen en el usuario alto interés, inmersión, satisfacción y efectividad en el aprendizaje, complementado con otros beneficios como el bajo costo en sistemas de este tipo en comparación con entrenamientos no virtuales (Román-Ibáñez et al., 2018).

\subsection{Hápticos, educación y RV}

La implementación de hápticos en realidad virtual se ha vuelto algo común, debido a que se genera una mejor experiencia de usuario y se le da un mayor valor al aprendizaje, tras complementar con hápticos las ventajas de retroalimentación generadas al usuario.

Un ejemplo de lo anterior puede verse en campos como la medicina ya que se requieren cirujanos con una formación sólida apoyada con la experiencia en cirugía general, con un simulador háptico para la cirugía de hueso (Morris et al., 2006) y cataratas (Agus et al., 2006), y en Mayorga (2018) donde se usa uno de los hardware más comunes en este tipo de proyectos: el Phanton Omni desarrollado por Sensable Technologies.

\section{Controles}

En el momento de construir el prototipo para educación/entrenamiento fue de vital importancia definir tanto los controles como su tipo, por esta razón surge la necesidad de elegir entre diferentes tipos de controles que son usados más comúnmente y que representan una mejor experiencia para el usuario. Dentro de la selección se encuentran los controles hápticos y los mandos tradicionales de consola.

\subsection{Controles Hápticos}

La palabra háptico en general se refiere a todo lo relacionado con el estudio del sentido del tacto y la capacidad del ser humano para interactuar por medio de este (Minogue \& Jones, 2006; Révész, 1950), sin embargo, en el contexto de interacción usuario-maquina se habla de dispositivos hápticos los cuales dan la posibilidad al usuario de interactuar en un medio virtual por medio del contacto físico, buscando así un intercambio simultaneo de información (Okamura et al., 2002). 
Los dispositivos hápticos se pueden clasificar como pasivos o activos (Hidalgo, 2011). Los pasivos son aquellos cuya interacción tangible determina retroalimentación para el usuario, por otro lado, los activos generan una retroalimentación propia basados en actuadores, sensores y software especializado para la interacción háptica.

Con respecto a lo antes planteado para este experimento se usan sensores Phidget (Phidgets Inc., 2016), definidos como dispositivos hápticos activos. La justificación para decidir incluir controles hápticos es que, a través del tacto activo, una persona no solo obtiene sensaciones, sino también información relevante que le ayuda a inferir y tomar decisiones incluso si no está utilizando los sentidos de la vista y el oído (Kennedy et al., 1992); algo bastante favorable para ambientes inmersivos, tal como se busca en Tauscher et al. (2019), donde las interacciones en entornos virtuales se mejoran con objetos reales.

\subsection{Mandos de consola}

Recientes estudios, demuestran como actualmente existe una excesiva utilización en la vida cotidiana de internet y videojuegos, lo que establece una gran cantidad de usuarios existentes que manipulan estas tecnologías, además de ser un tema de gran interés para la comunidad científica mundial (Chahín Pinzón \& Briñez, 2018).

Siendo el mercado de videojuegos altamente competitivo por la cantidad de usuarios existentes, es necesario generar acciones para demostrar valor y ser competitivo. Es por esta razón que los videojuegos han requerido de grandes inversiones que empresas líderes como Microsoft y Sony han realizado para garantizar que sus consolas sean usables, ergonómicas y generen satisfacción en sus usuarios (Dalgleish, 2018). Por esta razón se decide utilizar un control tradicional de Xbox One, por ser una de las consolas más populares a la fecha y su compatibilidad con los dispositivos de realidad virtual, complementado con estudios que demuestran la preferencia significativa para Xbox con respecto a otras consolas (Brown \& Mackenzie, 2013).

\section{Usabilidad}

Varios autores contextualizan el uso de la palabra usabilidad a interfaces gráficas definiéndola como una medida con la cual una herramienta o producto puede ser usada para lograr objetivos en un contexto específico, destacando tres dimensiones: eficacia, eficiencia y satisfacción (Finstad, 2010; Sik Lanyi et al., 2010).

Basados en los estudios realizados en Sik Lanyi et al. (2010) donde se mide usabilidad para juegos serios para estudiantes con discapacidad intelectual, se puede decir que la usabilidad en juegos es similar a la de software, diferenciándolas en que en los juegos se busca más una experiencia amigable con el usuario y que sea divertida. Además, incentivan que crezca la cantidad de investigación en realidad virtual para educación, lo que dio la oportunidad de generar un experimento que contribuya a las realidades de los entornos virtuales y que pueda implementar las dimensiones mencionadas anteriormente para medir su usabilidad, sin embargo, para este experimento solo son aplicables las dimensiones de eficiencia y satisfacción, ya que la efectividad está más ligada a medirse en el sistema en su totalidad (Sik Lanyi et al., 2010), de esta manera no se considera un punto relevante para la comparación de los controles.

\section{Prototipo}

El prototipo es una aplicación de realidad virtual diseñada para realizar tareas simples de entrenamiento en la manipulación general de una grúa. Para la escena de realidad virtual se usaron assets, texturas y materiales gratuitos o en algunos casos, construidos por autoría propia. Con respecto a la grúa, se trata de un tipo torre con polea y gancho que sujetan una base plana.

Complementando, el prototipo se encuentra disponible para Oculus Rift y fue desarrollada con el motor de videojuegos Unity en su versión 2018.3.0f2. Los dispositivos utilizados fueron:

- Sensores Phydget tipo touch, Referencia 1110. 
- Joystick Logitech Force30Pro.

- Ordenador Dell, S.O Windows 10.

- Oculus Rift.

- Control de Xbox One.

El ensamblaje de hápticos consistió en la transmisión de señales de los dispositivos de esta índole, por medio de conexión USB y las respectivas librerías para la manipulación de estas en Unity con C\#. Mientras que para el control tradicional se realizó el mapeo correspondiente, de tal manera que las funciones asignadas a cada botón fueran fáciles de entender para los usuarios. En la Figura 1, se muestran los dispositivos implementados para el prototipo de entrenamiento en la manipulación general de una grúa con realidad virtual. Se pueden observar como controles hápticos: un joystick y los sensores touch ubicados sobre un soporte que simula una caja de control, adicionalmente, como control tradicional un control de Xbox One también usado para simular los movimientos de vibración de la grúa.

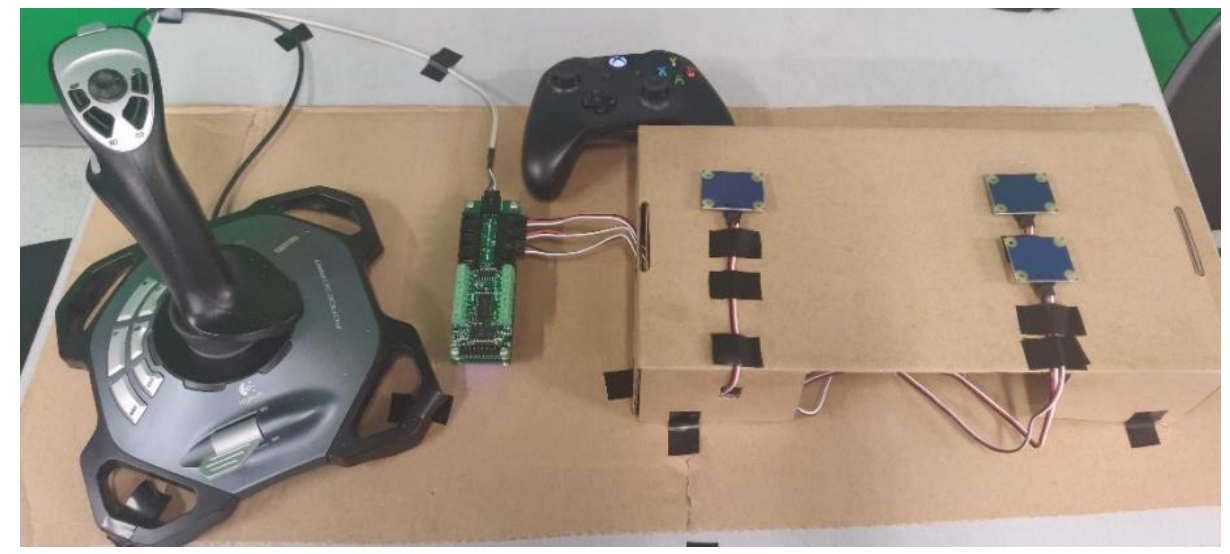

Figura 1. Ensamblaje

\subsection{Mapeo de controles}

Para los controles hápticos, el joystick permite movilidad de la base de grúa. En su movimiento horizontal permite su giro con pivote en su centro, mientras que el vertical el movimiento de la polea a lo largo del brazo de la grúa. El sensor ubicado en la parte izquierda permite el encendido y apagado de la grúa, los otros dos ubicados a la derecha permiten la manipulación de la carga de la polea, permitiendo subir y bajar la misma.

En el caso del control del Xbox el stick derecho realiza la misma funcionalidad del joystick, el botón "X" permite encender y apagar la grúa y los botones "Y" y "A" permiten subir y bajar la carga de la polea respectivamente.

\section{Pruebas}

En este apartado se describe la metodología y los artefactos de medición implantados para la medición de las tres dimensiones de usabilidad en el prototipo desarrollado.

\subsection{Metodología}

Con el propósito de facilitar el análisis y la toma de decisiones, se decide optar por un público objetivo de diez personas, las cuales son estudiantes hombres y mujeres que pueden encontrarse cursando tanto pregrado como posgrado en la Universidad de Los Andes, Colombia; es decir, sus edades varían entre los 18 y los 30 años aproximadamente y tienen conocimientos sobre realidad virtual. 
A continuación, se describen las instrucciones y recomendaciones dadas a los participantes para realizar las pruebas:

1. Explicación sobre controles hápticos, ¿qué son? Y que funcionalidad cumplen en el prototipo.

2. Se procede a iniciar la aplicación y colocar las gafas de realidad virtual al participante.

3. Se le pide ubicar el joystick con la mano izquierda y la caja de control con la derecha.

Posteriormente, estas son las tareas que se le solicitaron realizar a los participantes:

1. Encender la grúa.

2. Girar la grúa $90^{\circ}$ a la derecha.

3. Subir la carga ubicada en la parte inferior hasta que sea visible.

4. Acercar la carga a la cabina lo más posible.

5. Apague la grúa.

Finalmente, se reinicia la aplicación y se pide repetir los pasos anteriores con el control tradicional (ver Figura 2).

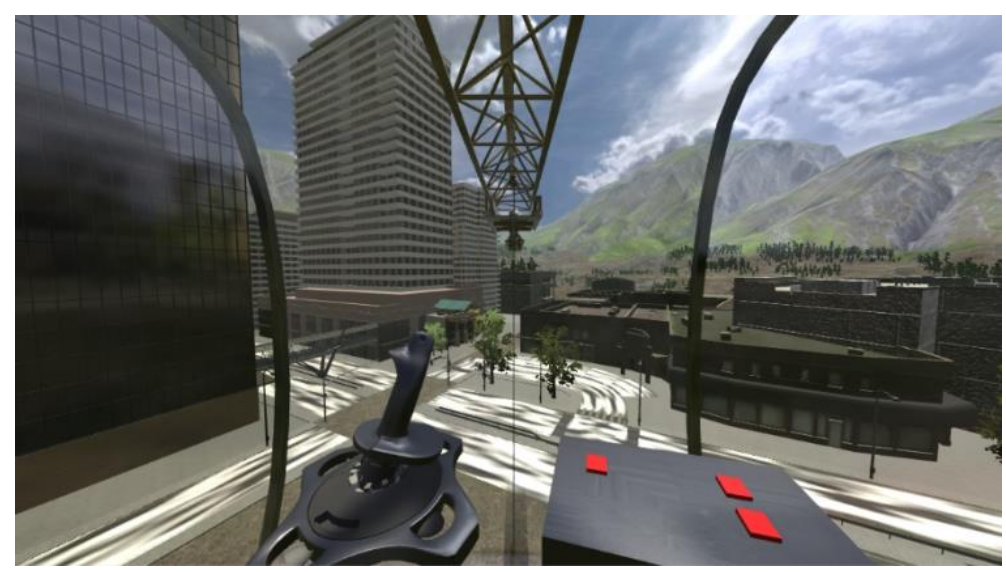

Figura 2. Visualización de una de las pruebas para la tarea dos, girar la grúa $90^{\circ}$ a la derecha

\subsection{Instrumentos}

Una vez finalizadas las pruebas, se solicitó a los participantes diligenciar encuestas. Se decide realizar preguntas tipo Likert basadas en la investigación de Sik Lanyi et al. (2010), donde se evalúa las tres dimensiones de usabilidad para medir la experiencia de los usuarios. Antes de diligenciar la encuesta todos los participantes aceptaron que al utilizar la aplicación y diligenciar el formulario, la información recopilada fuera usada para fines investigativos.

Tal como se realiza en (Medina \& Figueroa, 2018), las preguntas tipo Likert permiten por medio de una escala medir actitudes y conocer el grado de conformidad con algo, en este caso la usabilidad del prototipo en diferentes dimensiones. Para la etapa de pruebas, fue definido un rango que permitiera a los participantes tener un punto de vista claro sobre lo que opinan de la pregunta, donde el rango esta definido por (1) Totalmente en desacuerdo y (7) Totalmente de acuerdo, lo anterior aplicado a dos encuestas tanto para controles hápticos como para tradicionales con ocho preguntas cada una.

\section{Resultados y análisis de resultados}

La comparación de los controles tradicionales (izquierda) con los controles hápticos (derecha) por medio de las preguntas Likert realizadas a los diez participantes con el respectivo resultado se puede evidenciar en las Figuras 3 a 10: 


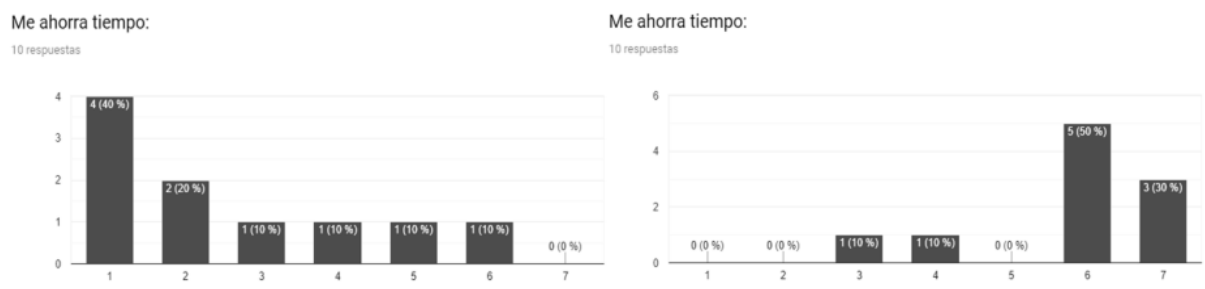

Figura 3. Respuestas obtenidas a la pregunta No. 1 eficiencia. ¿Me ahora tiempo?

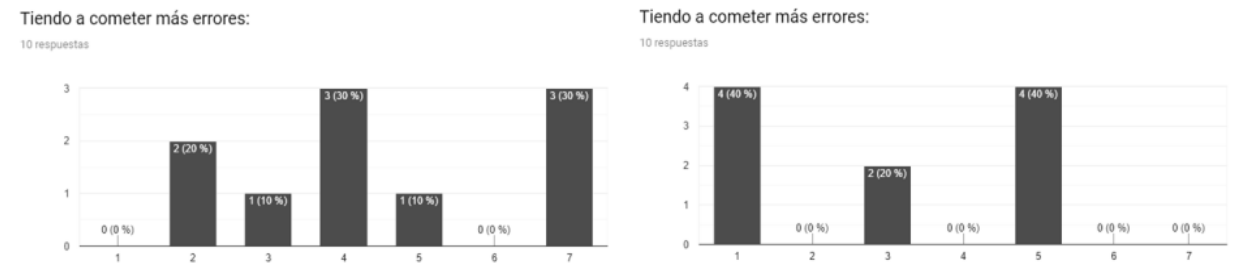

Figura 4. Respuestas obtenidas a la pregunta No. 2 eficiencia. ¿Tiendo a cometer más errores?

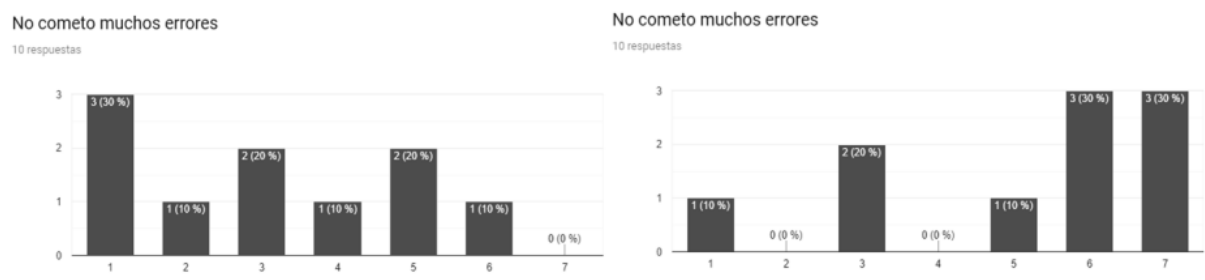

Figura 5. Respuestas obtenidas a la pregunta No. 3 eficiencia. ¿No comento muchos errores?

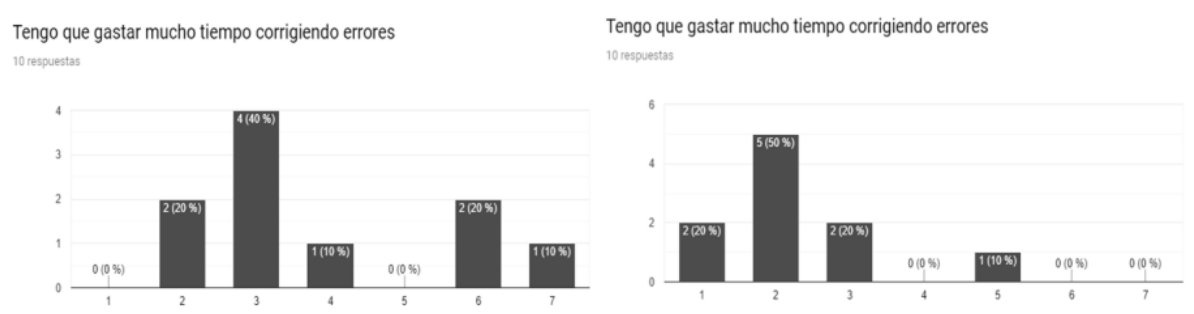

Figura 6. Respuestas obtenidas a la pregunta No. 4 eficiencia. ¿Tengo que gastar mucho tiempo corrigiendo errores?

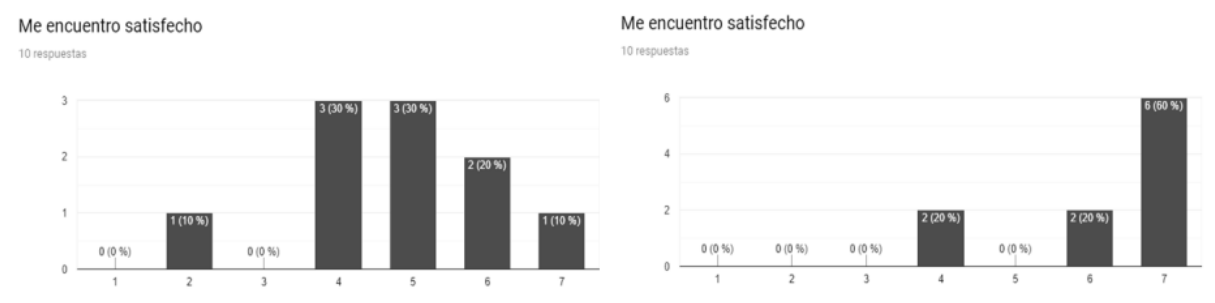

Figura 7. Respuestas obtenidas a la pregunta No. 5 satisfacción. ¿Me encuentro satisfecho?

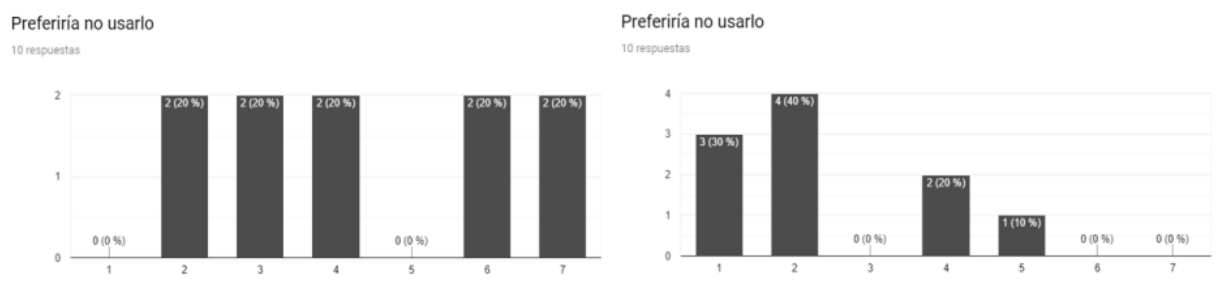

Figura 8. Respuestas obtenidas a la pregunta No. 6 satisfacción. ¿Preferiría no usarlo? 

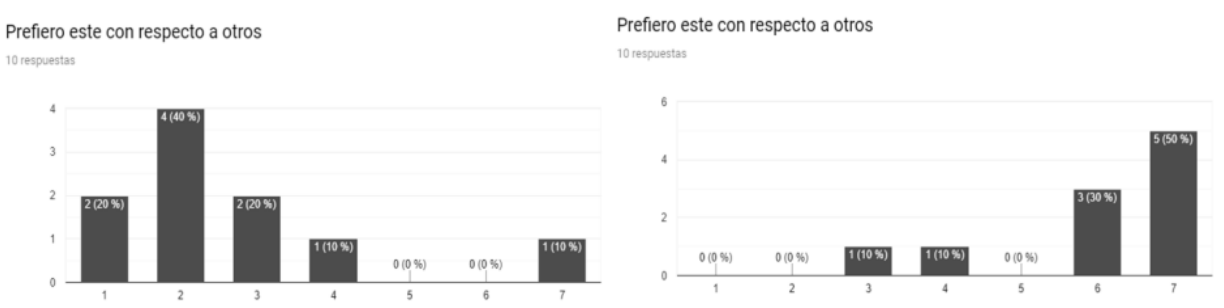

Figura 9. Respuestas obtenidas a la pregunta No. 7 satisfacción. ¿Prefiero este con respecto a otros?
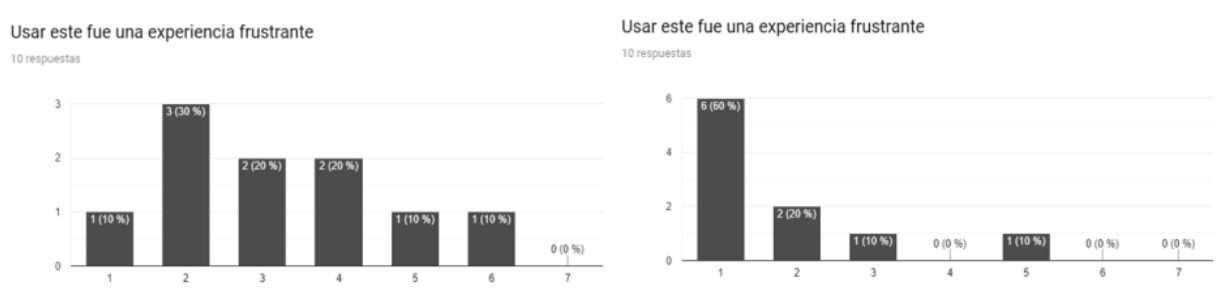

Figura 10. Respuestas obtenidas a la pregunta No. 8 satisfacción. ¿Usar este fue una experiencia frustrante?

Los anteriores resultados dan a entender una clara preferencia de la experiencia de los usuarios a usar controles del tipo hápticos a controles tradicionales, destacando dicha preferencia en términos de eficiencia y satisfacción. Lo que significa un mejor desempeño y experiencia para ellos realizando tareas de entrenamiento y educación con este tipo de controles.

\section{Conclusiones y trabajo futuro}

Posterior a la finalización de un prototipo inicial para educación/entrenamiento y de acuerdo con los resultados obtenidos en las encuestas, es válido afirmar que los controles de tipo hápticos para realidad virtual complementan mejor la experiencia del usuario debido a que los mismos los consideran más eficientes y satisfactorios.

Como trabajo futuro, se propone extender el proyecto para mejorar aún más la experiencia del usuario, interfaces, explorar nuevas tendencias que permitan innovar en la misma y profundizar con los controles hápticos para desarrollar nuevas experiencias y aplicaciones. Así mismo abordar nuevos campos en los que se puedan aplicar este tipo de proyectos y que cumplan su función para educación/entrenamiento.

\section{Declaración de conflicto de intereses}

Los autores declaran no tener conflicto de intereses con respecto a la investigación, autoría o publicación de este artículo.

\section{Referencias}

Agus, M., Gobbetti, E., Zanetti, G., \& Zorcolo, A. (2006). Real Time Simulation of Phaco-emulsification for Cataract Surgery Training. Real-time Cataract Surgery Simulation for Training. En C. Mendoza \& I. Navazo (Eds.), 3rd Workshop in Virtual Reality Interactions and Physical Simulation "VRIPHYS" (2006). The Eurographics Association. https://doi.org/10.2312/PE/vriphys/vriphys06/091-100

Al-Shamaileh, N. (2014). Cognitive Motivational Learning of the Students of the Faculty of Medicine at the University of Jordan. Gifted and Talented International, 29(1-2), 99-112. https://doi.org/10.1080/15332276.2014.11678433

Boejen, A., \& Grau, C. (2011). Virtual reality in radiation therapy training. Surgical Oncology, 20(3), 185-188. https://doi.org/10.1016/j.suronc.2010.07.004

Boychenko, K. V. (2017). Virtual reality as the tool of interactive architecture. Problems of modern science and 
education, 90. https://doi.org/10.20861/2304-2338-2017-90-004

Brown, M. A., \& Mackenzie, I. S. (2013). Evaluating Video Game Controller Usability as Related to User Hand Size. Proceedings of the International Conference on Multimedia and Human Computer Interaction. http://citeseerx.ist.psu.edu/viewdoc/summary?doi=10.1.1.417.7071

Chahín Pinzón, N., \& Briñez, B. L. (2018). Propiedades psicométricas del Cuestionario de Adicción a Internet y a los Videojuegos para Adolescentes. Universitas Psychologica, 17(4), 1-13. https://doi.org/10.11144/Javeriana.upsy17-4.ppca

Dalgleish, M. (2018). There are no universal interfaces: how asymmetrical roles and asymmetrical controllers can increase access diversity. $G|A| M \mid E$ Games as Art, Media, Entertainment, 7. https:/www.gamejournal.it/wpcontent/uploads/2019/08/GAME 07 SpecialNeeds Dalgleish.pdf

Finstad, K. (2010). The Usability Metric for User Experience. Interacting with Computers, 22(5), $323-327$. https://doi.org/10.1016/j.intcom.2010.04.004

Hidalgo, G. A. (2011). Supervised learning for haptics texture classification using fourier analysis [Instituto Tecnológico y de Estudios Superiores de Monterrey]. https://repositorio.tec.mx/handle/11285/570646

Kennedy, J. M., Gabias, P., \& Heller, M. A. (1992). Space, haptics and the blind. Geoforum, 23(2), $175-189$. https://doi.org/10.1016/0016-7185(92)90015-V

Li, L., Zhang, M., Xu, F., \& Liu, S. (2005). ERT-VR: an immersive virtual reality system for emergency rescue training. Virtual Reality, 8(3), 194-197. https://doi.org/10.1007/s10055-004-0149-6

Ma, M., Jain, L. C., \& Anderson, P. (2014). Virtual, Augmented Reality and Serious Games for Healthcare 1. Springer, Berlin, Heidelberg. https://doi.org/10.1007/978-3-642-54816-1

Mayorga, D. R. (2018). Simulador multimodal de entrenamiento en reducción de fracturas como módulo de residencia. Universidad de los Andes.

Medina, F., \& Figueroa, P. (2018). Prototype of a Serious Game for Peace Construction in a Colombian Context. 2018 XLIV Latin American Computer Conference (CLEI), 328-333. https://doi.org/10.1109/CLEI.2018.00047

Minogue, J., \& Jones, M. G. (2006). Haptics in Education: Exploring an Untapped Sensory Modality. Review of Educational Research, 76(3), 317-348. https://doi.org/10.3102/00346543076003317

Morris, D., Sewell, C., Barbagli, F., Salisbury, K., Blevins, N. H., \& Girod, S. (2006). Visuohaptic simulation of bone surgery for training and evaluation. IEEE Computer Graphics and Applications, 26(6), 48-57. https://doi.org/10.1109/MCG.2006.140

Okamura, A. M., Richard, C., \& Cutkosky, M. R. (2002). Feeling is Believing: Using a Force-Feedback Joystick to Teach Dynamic Systems. Journal of Engineering Education, 91(3), 345-349. https://doi.org/10.1002/j.21689830.2002.tb00713.x

Phidgets Inc. (2016). Phidgets Inc. - Products for USB Sensing and Control. https://www.phidgets.com/?

Révész, G. (1950). Experimental study in abstraction in monkeys. Longmans, Green. https://psycnet.apa.org/record/1951-03367-000

Román-Ibáñez, V., Pujol-López, F., Mora-Mora, H., Pertegal-Felices, M., \& Jimeno-Morenilla, A. (2018). A LowCost Immersive Virtual Reality System for Teaching Robotic Manipulators Programming. Sustainability, 10(4), 1102. https://doi.org/10.3390/su10041102

Sik Lanyi, C., Brown, D. J., Standen, P., Lewis, J., \& Butkute, V. (2010). User Interface Evaluation of Serious Games for Students with Intellectual Disability. En Lecture Notes in Computer Science (including subseries Lecture Notes in Artificial Intelligence and Lecture Notes in Bioinformatics): Vol. 6179 LNCS (Número PART 1, pp. 227-234). https://doi.org/10.1007/978-3-642-14097-6_37

Tauscher, J.-P., Schottky, F. W., Grogorick, S., Bittner, P. M., Mustafa, M., \& Magnor, M. (2019). Immersive EEG: Evaluating Electroencephalography in Virtual Reality. 2019 IEEE Conference on Virtual Reality and $3 D$ User Interfaces (VR), 1794-1800. https://doi.org/10.1109/VR.2019.8797858

Wang, G.-X., \& Li, L. (2012). Virtual reality exposure therapy of anxiety disorders. Advances in Psychological Science, 20(8), 1277-1286. http://journal.psych.ac.cn/xlkxjz/EN/abstract/abstract2584.shtml

Watanuki, K., \& Kojima, K. (2006). Virtual Reality Based Job Training for Advanced Manufacturing Skills. The International Conference on Business \& Technology Transfer, 2006.3, 134-139. https://doi.org/10.1299/jsmeicbtt.2006.3.0 134

Yilmaz, B., \& Goken, M. (2016). Virtual reality (VR) technologies in education of industrial design. New Trends and Issues Proceedings on Humanities and Social Sciences, 2(1), 498-503. https://doi.org/10.18844/gjhss.v2i1.336

\section{Sobre los autores}

Pablo Alejandro Figueroa

Profesor en el Departamento de Ingeniería de Sistemas y Computación en la Universidad de los Andes. Magíster en Ingeniería de Sistemas y Computación, Universidad de los Andes, Colombia. Ph.D. en Ciencias 
de la Computación, Universidad de Alberta, Canadá. Maneja líneas de investigación en realidad virtual y aumentada, y en desarrollo de videojuegos.

\section{Fabián Medina Cortés}

Magister en Ingeniería de software de la Universidad de los Andes de Bogotá, Colombia; Ingeniero de Sistemas de la Universidad El Bosque de Bogotá, Colombia. Participación en grupos de investigación OSIRIS e Imagine. Desempeño profesional en investigación, desarrollo de software e innovación tecnológica en áreas como realidad virtual, realidad aumentada, IoT y automatización para el sector educativo, retail e inmobiliario. 